CERN-TH.6145/91

LPTENS 91/17

\title{
W-ALGEBRAS FROM CHERN-SIMONS THEORY
}

\author{
Adel Bilal \\ Theory Division, CERN, 1211 Geneva 23, Switzerland \\ and \\ Laboratoire de Physique Théorique de l'Ecole Normale Supérieure, \\ 24 rue Lhomond, 75231 Paris Cedex 05, France
}

\begin{abstract}
We show that the physical state conditions of $S l(3, \mathbf{R})$ Chern-Simons theory are equivalent to the Ward identities of the $W_{3}^{(2)}$-algebra. More precisely, choosing a specific polarization for quantizing the Chern-Simons theory, we can solve explicitly part of the Chern-Simons physical state conditions, while the remaining ones reduce to the Ward identities satisfied by the conformal blocks of the $W_{3}^{(2)}$-algebra extended conformal field theory (implying the OPEs). It is an obvious conjecture that a different choice of polarization leads to the Ward identities of $W_{3} \equiv W_{3}^{(1)}$ and that these relations can be generalized to $S l(n, \mathbf{R})$ Chern-Simons and $W_{n}$.
\end{abstract}




\section{Introduction}

$W$-algebras are by now an important concept in theoretical physics. While they were originally found [1] in the context of conformal field theory as an extension of the Virasoro algebra by a spin-3 primary field, they appear now to be related to many other areas of theoretical physics, such as cosets of affine Lie algebras [2], gauged WZWmodels [3,4], reductions of the KP-hierarchy [5,6], Toda field theories [7], integrable IRF lattice models [8] and, more recently, matrix models of random surfaces [9].

The Virasoro algebra has a clear geometrical meaning as related to the diffeomorphisms and the geometry of Riemann surfaces. The associated conformal Ward identity can be interpreted [10] as expressing the compatibility of complex structures (parametrized by the Beltrami differential $\mu$ ) and projective structures (parametrized by the stress tensor $T$ ) on a Riemann surface. An attempt to obtain a similar interpretation for the Ward identities of the $W_{n}$-algebra was only partially successful [10].

An important step towards the understanding of the origin of $W$-algebras was the realization that the full set of their Ward identities (implying all OPEs) can be obtained by a certain Hamiltonian reduction of two-dimensional $S l(n)$-connections [10]. In this formalism the Ward identities appear as zero-curvature conditions on the reduced symplectic manifold. This is a very powerful approach but one might find the concept of Hamiltonian reduction and in particular the choice of the reducing symmetry group somewhat unmotivated. The goal of the present paper is to provide a more straightforward (although algebraically more lengthy) alternative.

Some time ago, H. Verlinde has shown that the physical state conditions arising in the quantization of the $S l(2, \mathbf{R})$ Chern-Simons gauge theory are equivalent to the conformal Ward identity satisfied by the conformal blocks of conformal field theory. It was a natural speculation that one should obtain the Ward identities of $W_{n}$-algebras when replacing $S l(2, \mathbf{R})$ by $S l(n, \mathbf{R})$.

However, since there are several inequivalent embeddings of $S l(2)$ into $S l(n)$ [12] corresponding to inequivalent Hamiltonian reductions of 2D $S l(n)$-gauge connections, there are several different $W_{n}$-algebras, labelled $W_{n}^{(1)}, W_{n}^{(2)}, \ldots W_{n}^{(n-1)}$. In this notation, the standard well-known $W_{n}$-algebras are denoted $W_{n}^{(1)}$. For example, $W_{3} \equiv W_{3}^{(1)}$ contains the stress tensor $T$ and a spin-3 field $W$, while the $W_{3}^{(2)}$-algebra contains, besides the stress tensor $T$, a spin-1 field $U$ and two bosonic spin- $\frac{3}{2}$ fields $G^{ \pm}$. Due to the bosonic nature of the latter, their OPE contains, in contrast to the $N=2$ superconformal algebra, a non-linear $U^{2}$-term. This algebra has been investigated in ref. 13. 
One thus expects that different choices of polarization in the quantization of the $S l(n)$ Chern-Simons theory will lead to different $W_{n}$-algebras. Since different polarizations are related by functional Laplace (Fourier) transformations, the $W_{n}^{(l)}$-algebras for different $l$ (but same $n$ ) could be viewed as equivalent up to Laplace (Fourier) transformations. In this paper we will only work out the simplest case $n=3$. Our choice of polarization will lead to the Ward identities of the $W_{3}^{(2)}$-algebra. The case of $W_{3}^{(1)} \equiv W_{3}$ and that of general $n$ will be described elsewhere.

The outline of this paper is the following: we first recall some simple facts about Chern-Simons (CS) theory, and, for the sake of being pedagogical, repeat the computation of ref. 11 leading from $S l(2, \mathbf{R})$ CS to the conformal Ward identity. Then, we show how the $S l(3, \mathbf{R})$ CS theory leads to the Ward identities of $W_{3}^{(2)}$.

\section{Some general facts about CS theory, and the conformal Ward identity from $S l(2, \mathbf{R}) \mathbf{C S}$}

Consider the Chern-Simons gauge theory with action

$$
S_{\mathrm{CS}}=\frac{k}{2 \pi i} \int_{M} \operatorname{tr}\left(A D A+\frac{2}{3} A^{3}\right)
$$

where $D$ denotes the exterior derivative in $2+1$ dimensions and $A(x)$ is a 3 -form with values in some Lie algebra $g$ of some Lie group $G$. Note that we will be interested in non-compact $G$, and hence $k$ will not be quantized. The 3 -manifold $M$ is supposed to be of the form $M=\Sigma \times \mathbf{R}, \Sigma$ being some Riemann surface, and $x=(z, \bar{z}, t)$. Now write

$$
A=a+A_{0} \mathrm{~d} t, \quad D=\mathrm{d}+\mathrm{d} t \partial_{t}
$$

with $a \equiv a(z, \bar{z}, t)=a_{z} \mathrm{~d} z+a_{\bar{z}} \mathrm{~d} \bar{z}$ a 2 -form on $\Sigma$ (depending also on $\left.t\right)$ and $\mathrm{d}$ the exterior derivative on $\Sigma$. Upon inserting this into the action (1) we get

$$
S_{\mathrm{CS}}=\frac{k}{2 \pi i} \int \mathrm{d} t \int_{\Sigma} \operatorname{tr}\left[a \wedge \partial_{t} a+2 A_{0}(\mathrm{~d} a+a \wedge a)\right]
$$

We see that $A_{0}$ acts as a Lagrange multiplier enforcing the constraints

$$
F(a) \equiv \mathrm{d} a+a \wedge a=0,
$$

while the first term (" $p \dot{q}$-term") equals $\frac{k}{\pi i} \int \mathrm{d} t \int_{\Sigma} \mathrm{d}^{2} z \operatorname{tr} a_{z} \partial_{t} a_{\bar{z}}$ and thus tells us that 
(at any fixed $t$ ) the symplectic structure is given by

$$
\left\{a_{\bar{z}}^{a}(z), a_{z}^{b}\left(z^{\prime}\right)\right\}=\frac{\pi i}{k} \delta^{a b} \delta^{(2)}\left(z-z^{\prime}\right)
$$

(provided one chooses a hermitian basis, $a=\sum a^{a} T^{a}, \operatorname{tr} T^{a} T^{b}=\delta^{a b}$ ).

Quantization involves the replacement $i\{,\} \rightarrow[$, ] and a choice of polarization: the Hilbert space is given by the space of functionals of a maximally commuting subset of the fields $a_{z}^{a}, a_{\bar{z}}^{a}$. One might e.g. choose all $a_{z}^{a}$, or all $a_{\bar{z}}^{a}$, but any "mixed" choice is equally valid. This is just like in ordinary quantum mechanics where one can choose to work in the coordinate-representation or in the momentum-representation or use one of them for certain dimensions and the other for the remaining ones. In any case, one can always change polarization by a Fourier (Laplace) transformation. We also have to deal with the constraints $F(a)=0$. We choose to quantize the theory first in the large phase space given by $(5)$ and then select the physical Hilbert space of physical states by requiring the constraints to hold as operator equations $F(a) \Psi=0$ on the physical states.

Let us now, following ref. 11 , discuss the case $g=S l(2, \mathbf{R})$ and show how it leads to the conformal Ward identity. Parametrize the $S l(2, \mathbf{R})$-valued 2 -form $a$ as

$$
a=\left(\begin{array}{cc}
\frac{1}{2} \omega & \frac{1}{\sqrt{2}} e^{+} \\
\frac{1}{\sqrt{2}} e^{-} & -\frac{1}{2} \omega
\end{array}\right)
$$

Then

$$
F(a)=\left(\begin{array}{cc}
\frac{1}{2}\left(\mathrm{~d} \omega+e^{+} \wedge e^{-}\right) & \frac{1}{\sqrt{2}}\left(\mathrm{~d} e^{+}+\omega \wedge e^{+}\right) \\
\frac{1}{\sqrt{2}}\left(\mathrm{~d} e^{-}-\omega \wedge e^{-}\right) & -\frac{1}{2}\left(\mathrm{~d} \omega+e^{+} \wedge e^{-}\right)
\end{array}\right)
$$

and we have

$$
\frac{k}{2 \pi i} \int a \wedge \partial_{t} a=\frac{k}{2 \pi i} \int \mathrm{d}^{2} z\left(\omega_{z} \wedge \partial_{t} \omega_{\bar{z}}+e_{z}^{+} \wedge \partial_{t} e_{\bar{z}}^{-}-e_{\bar{z}}^{+} \wedge \partial_{t} e_{z}^{-}\right)
$$

Hence

$$
\left[\omega_{z}(z), \omega_{\bar{z}}\left(z^{\prime}\right)\right]=\left[e_{z}^{-}(z), e_{\bar{z}}^{+}\left(z^{\prime}\right)\right]=-\left[e_{\bar{z}}^{-}(z), e_{z}^{+}\left(z^{\prime}\right)\right]=\frac{2 \pi}{k} \delta^{(2)}\left(z-z^{\prime}\right)
$$

One chooses as maximally commuting subset the fields $\omega_{z}, e_{z}^{+}$and $e_{\bar{z}}^{+}$. With this 
polarization

$$
\omega_{\bar{z}}(z)=-\frac{2 \pi}{k} \frac{\delta}{\delta \omega_{z}(z)}, \quad e_{\bar{z}}^{-}(z)=-\frac{2 \pi}{k} \frac{\delta}{\delta e_{z}^{+}(z)}, \quad e_{z}^{-}(z)=+\frac{2 \pi}{k} \frac{\delta}{\delta e_{\bar{z}}^{+}(z)}
$$

acting on wave functionals $\Psi\left(\omega_{z}, e_{z}^{+}, e_{\bar{z}}^{+}\right)$. The constraints $F(a) \Psi=0$ become three operator constraints

$$
\mathcal{C}_{a} \Psi\left(\omega_{z}, e_{z}^{+}, e_{\bar{z}}^{+}\right)=0, \quad a=0, \pm
$$

with

$$
\begin{aligned}
& \mathcal{C}_{+}=\partial e_{\bar{z}}^{+}-\vec{\partial} e_{z}^{+}+\omega_{z} e_{\bar{z}}^{+}+\frac{2 \pi}{k} e_{z}^{+} \frac{\delta}{\delta \omega_{z}} \\
& \mathcal{C}_{0}=\partial \frac{\delta}{\delta \omega_{z}}+\frac{k}{2 \pi} \bar{\partial} \omega_{z}+e_{z}^{+} \frac{\delta}{\delta e_{z}^{+}}+e_{\bar{z}}^{+} \frac{\delta}{\delta e_{\bar{z}}^{+}} \\
& \mathcal{C}_{-}=\partial \frac{\delta}{\delta e_{z}^{+}}+\bar{\partial} \frac{\delta}{\delta e_{\bar{z}}^{+}}-\omega_{z} \frac{\delta}{\delta e_{z}^{+}}+\frac{2 \pi}{k} \frac{\delta}{\delta \omega_{z}} \frac{\delta}{\delta e_{\bar{z}}^{+}}
\end{aligned}
$$

One has to solve these three functional differential equations. In fact, one is able to solve $\mathcal{C}_{+} \Psi=0$ and $\mathcal{C}_{0} \Psi=0$ by a clever change of variables, while the remaining constraint $\mathcal{C}_{-} \Psi=0$ becomes equivalent to the conformal Ward identity. Parametrize

$$
e_{z}^{+}=e^{\varphi}, e_{\bar{z}}^{+}=e^{\varphi} \mu
$$

where $e^{\varphi}=\left(e^{\varphi}\right)_{z}$ and $\mu=\mu_{\bar{z}}^{z}$. Thus $e^{+}=e^{\varphi}(\mathrm{d} z+\mu \mathrm{d} \bar{z})$ which is very natural if we interpret $e^{+}$as zweibein, $\varphi$ as (chiral) Liouville field and $\mu$ as Beltrami differential. (To simplify the notation, in the following we will simply write $\omega$ instead of $\omega_{z}$.) The constraint operators now read

$$
\begin{aligned}
e^{-\varphi} \mathcal{C}_{+} & =\frac{2 \pi}{k} \frac{\delta}{\delta \omega}+\mu \omega+\mu \partial \varphi+\partial \mu-\bar{\partial} \varphi \\
\mathcal{C}_{0} & =\frac{\delta}{\delta \varphi}+\partial \frac{\delta}{\delta \omega}+\frac{k}{2 \pi} \bar{\partial} \omega \\
e^{\varphi} \mathcal{C}_{-} & =(\bar{\partial}-\mu \partial-2 \partial \mu) \frac{\delta}{\delta \mu}+(\partial-\partial \varphi-\omega) \frac{\delta}{\delta \varphi}+e^{-\varphi} \mathcal{C}_{+} \frac{\delta}{\delta \mu}
\end{aligned}
$$

We can solve $\mathcal{C}_{+} \Psi=0$ by

$$
\Psi\left(\omega_{z}, e_{z}^{+}, e_{\bar{z}}^{+}\right) \equiv \Psi(\omega, \varphi, \mu)=\exp \left(-\frac{k}{2 \pi} S_{1}[\omega, \varphi, \mu]\right) \Psi_{1}(\varphi, \mu)
$$

with

$$
S_{1}[\omega, \varphi, \mu]=\int \mathrm{d}^{2} z\left[\frac{1}{2} \mu \omega^{2}-\omega(\bar{\partial} \varphi-\mu \partial \varphi-\partial \mu)\right] .
$$

Since $\Psi_{1}(\varphi, \mu)=\Psi(\omega=0, \varphi, \mu)$, the constraints $\mathcal{C}_{0}$ and $\mathcal{C}_{-}$on $\Psi$ become new con- 
straints $\mathcal{C}_{0}^{(1)}$ and $\mathcal{C}_{-}^{(1)}$ on $\Psi_{1}$ that do not depend on $\omega$ anymore. Indeed, one finds

$$
\begin{aligned}
\mathcal{C}_{0}^{(1)} & =\frac{\delta}{\delta \varphi}+\frac{k}{2 \pi} \partial(\bar{\partial} \varphi-\mu \partial \varphi-\partial \mu) \\
\mathcal{C}_{\sim}^{(1)} & =(\bar{\partial}-\mu \partial-2 \partial \mu) \frac{\delta}{\delta \mu}+(\partial-\partial \varphi) \frac{\delta}{\delta \varphi}
\end{aligned}
$$

We remark that in passing from $\mathcal{C}_{-}$to $\mathcal{C}_{-}^{(1)}$ one obtains a term $\sim \frac{\delta}{\delta \omega(z)} \frac{\delta}{\delta \mu(z)} S_{1}$, diverging like some combination of $\delta(0)$ and $\delta^{\prime}(0)$. This divergence should be eliminated by a proper regularization (point-splitting) and renormalization (probably of the coupling constant $k$ ). Here, we will simply drop it.

Again, $\mathcal{C}_{0}^{(1)} \Psi_{1}=0$ is easily solved by

$$
\Psi_{1}(\varphi, \mu)=\exp \left(-\frac{k}{2 \pi} S_{2}[\varphi, \mu]\right) \Psi_{2}(\mu)
$$

with

$$
S_{2}[\varphi, \mu]=\int \mathrm{d}^{2} z\left[-\frac{1}{2} \partial \varphi \bar{\partial} \varphi+\frac{1}{2} \mu(\partial \varphi)^{2}-\mu \partial^{2} \varphi\right]
$$

which is a chiral version of the Liouville action [11]. Finally, $\mathcal{C}_{-}^{(1)} \Psi_{1}=0$ translates into $\mathcal{C}_{-}^{(2)} \Psi_{2}=0$ :

$$
\left[(\bar{\partial}-\mu \partial-2 \partial \mu) \frac{\delta}{\delta \mu}+\frac{k}{2 \pi} \partial^{3} \mu\right] \Psi_{2}(\mu)=0 .
$$

This is nothing but the conformal Ward identity for a conformal block $\Psi_{2}(\mu)$ in a CFT with central charge $c=6 k$. Thus one arrives at the conclusion that

$$
\Psi(\omega, \varphi, \mu)=\exp \left(-\frac{k}{2 \pi}\left(S_{1}[\omega, \varphi, \mu]+S_{2}[\varphi, \mu]\right)\right) \Psi_{2}(\mu)
$$

and $\Psi$ satisfies the physical state conditions $F(a) \Psi=0$ of the $S l(2, \mathbf{R})$ CS theory if and only if $\Psi_{2}$ satisfies the conformal Ward identity (20). In this sense, both conditions are equivalent. 


\section{From $S l(3, \mathbf{R})$ CS theory to $W_{3}^{(2)}$}

We will now repeat the previous exercise, replacing $S l(2, \mathbf{R})$ by $S l(3, \mathbf{R})$. We parametrize

$$
a=\left(\begin{array}{ccc}
\frac{1}{2}(\sigma+\omega) & \frac{1}{\sqrt{2}} f^{+} & \frac{1}{\sqrt{2}} g^{++} \\
\frac{1}{\sqrt{2}} f^{-} & -\sigma & \frac{1}{\sqrt{2}} e^{+} \\
\frac{1}{\sqrt{2}} g^{--} & \frac{1}{\sqrt{2}} e^{-} & \frac{1}{2}(\sigma-\omega)
\end{array}\right)
$$

Then

$$
F(a) \equiv \mathrm{d} a+a \wedge a=\left(\begin{array}{ccc}
\frac{1}{2}\left(\mathcal{C}_{5}+\mathcal{C}_{4}\right) & \frac{1}{\sqrt{2}} \mathcal{C}_{3} & \frac{1}{\sqrt{2}} \mathcal{C}_{1} \\
\frac{1}{\sqrt{2}} \mathcal{C}_{6} & -\mathcal{C}_{5} & \frac{1}{\sqrt{2}} \mathcal{C}_{2} \\
\frac{1}{\sqrt{2}} \mathcal{C}_{8} & \frac{1}{\sqrt{2}} \mathcal{C}_{7} & \frac{1}{2}\left(\mathcal{C}_{5}-\mathcal{C}_{4}\right)
\end{array}\right)
$$

We choose the following polarization: the wave functional $\Psi$ depends on $e \equiv e_{z}^{+}, \vec{e} \equiv e_{\bar{z}}^{+}$, $f \equiv f_{z}^{+}, \bar{f} \equiv f_{\bar{z}}^{+}, g \equiv g_{z}^{++}, \bar{g} \equiv g_{\bar{z}}^{++}, \omega \equiv \omega_{z}$ and $\sigma \equiv \sigma_{z}{ }^{\star}{ }^{*}$ Then

$$
\begin{gathered}
\omega_{\bar{z}}=\partial_{\omega}, \sigma_{\bar{z}}=\frac{1}{3} \partial_{\sigma}, e_{z}^{-}=-\partial_{\bar{e}}, e_{\bar{z}}^{-}=\partial_{e} \\
f_{z}^{-}=-\partial_{\bar{f}}, f_{\bar{z}}^{-}=\partial_{f}, g_{z}^{--}=-\partial_{\bar{g}}, g_{\bar{z}}^{--}=\partial_{g}
\end{gathered}
$$

where $\partial_{f}$ etc is shorthand for $\left(-\frac{2 \pi}{k}\right) \frac{\delta}{\delta f}$ etc.

Upon inserting (24) into (23) we get

$$
\begin{aligned}
& \mathcal{C}_{1}=\partial \bar{g}-\bar{\partial} g+\omega \bar{g}-g \partial_{\omega}+\frac{1}{\sqrt{2}}(f \bar{e}-\bar{f} e) \\
& \mathcal{C}_{2}=\partial \bar{e}-\bar{\partial} e-\frac{3}{2} \sigma \bar{e}+\frac{1}{2} e \partial_{\sigma}+\frac{1}{2} \omega \bar{e}-\frac{1}{2} e \partial_{\omega}-\frac{1}{\sqrt{2}}\left(g \partial_{f}+\bar{g} \partial_{\bar{f}}\right) \\
& \mathcal{C}_{3}=\partial \bar{f}-\bar{\partial} f+\frac{3}{2} \sigma \bar{f}-\frac{1}{2} f \partial_{\sigma}+\frac{1}{2} \omega \bar{f}-\frac{1}{2} f \partial_{\omega}+\frac{1}{\sqrt{2}}\left(g \partial_{e}+\bar{g} \partial_{\bar{e}}\right) \\
& \mathcal{C}_{4}=\partial \partial_{\omega}-\bar{\partial} \omega+\frac{1}{2}\left(e \partial_{e}+\bar{e} \partial_{\bar{e}}+f \partial_{f}+\bar{f} \partial_{\bar{f}}\right)+g \partial_{g}+\bar{g} \partial_{\bar{g}} \\
& \mathcal{C}_{5}=\frac{1}{3} \partial \partial_{\sigma}-\bar{\partial} \sigma+\frac{1}{2}\left(f \partial_{f}+\bar{f} \partial_{\bar{f}}-e \partial_{e}-\bar{e} \partial_{\bar{e}}\right) \\
& \mathcal{C}_{6}=\partial \partial_{f}+\bar{\partial} \partial_{\bar{f}}-\frac{1}{2} \omega \partial_{f}-\frac{1}{2} \partial_{\omega} \partial_{\bar{f}}-\frac{3}{2} \sigma \partial_{f}-\frac{1}{2} \partial_{\sigma} \partial_{\bar{f}}+\frac{1}{\sqrt{2}}\left(e \partial_{g}+\bar{e} \partial_{\bar{g}}\right) \\
& \mathcal{C}_{7}=\partial \partial_{e}+\bar{\partial} \partial_{\bar{e}}-\frac{1}{2} \omega \partial_{e}-\frac{1}{2} \partial_{\omega} \partial_{\bar{e}}+\frac{3}{2} \sigma \partial_{e}+\frac{1}{2} \partial_{\sigma} \partial_{\bar{e}}-\frac{1}{\sqrt{2}}\left(f \partial_{g}+\bar{f} \partial_{\bar{g}}\right) \\
& \mathcal{C}_{8}=\partial \partial_{g}+\bar{\partial} \partial_{\bar{g}}-\omega \partial_{g}-\partial_{\omega} \partial_{\bar{g}}+\frac{1}{\sqrt{2}}\left(\partial_{e} \partial_{\bar{f}}-\partial_{\bar{e}} \partial_{f}\right) .
\end{aligned}
$$

We have to solve $F \Psi=0$, i.e. $\mathcal{C}_{a} \Psi=0, a=1, \ldots 8$. First, we can easily solve $\mathcal{C}_{1} \Psi=0$

* Note that in eq. (22) $\sigma$ and $\omega$ denoted 2-forms while from now on they denote $\sigma_{z}$ and $\omega_{z}$. This should lead to no confusion and avoid too clumpsy notation. 
since $\mathcal{C}_{1}$ only involves one functional derivative. We set

$$
\Psi(\omega, \sigma, e, \bar{e}, f, \bar{f}, g, \bar{g})=\exp \left(-\frac{k}{2 \pi} S_{1}\right) \Psi_{1}(\sigma, e, \bar{e}, f, \bar{f}, g, \bar{g})
$$

with

$$
S_{1}=\int \mathrm{d}^{2} z\left(\frac{\bar{g}}{2 g} \omega^{2}+\omega \Omega\right), \Omega=\frac{1}{g}\left(\partial \bar{g}-\bar{\partial} g+\frac{f \bar{e}-\vec{f} e}{\sqrt{2}}\right) .
$$

$\Psi_{1}$ does no longer depend on $\omega$, and the constraints $\mathcal{C}_{a} \Psi=0, a=1, \ldots 8$ translate into new constraints $\mathcal{C}_{a}^{(1)} \Psi_{1}=0, a=2, \ldots 8$ with the $\mathcal{C}_{a}^{(1)}$ independent of $\omega, \partial_{\omega}^{\dagger}$ Explicitly we have

$$
\begin{aligned}
& \mathcal{C}_{2}^{(1)}=\partial \bar{e}-\bar{\partial} e-\frac{3}{2} \sigma \bar{e}+\frac{1}{2} e \partial_{\sigma}-\frac{1}{2} e \Omega-\frac{1}{\sqrt{2}}\left(g \partial_{f}+\bar{g} \partial_{\bar{f}}\right) \\
& \mathcal{C}_{3}^{(1)}=\partial \bar{f}-\bar{\partial} f+\frac{3}{2} \sigma \bar{f}-\frac{1}{2} f \partial_{\sigma}-\frac{1}{2} f \Omega+\frac{1}{\sqrt{2}}\left(g \partial_{e}+\bar{g} \partial_{\bar{e}}\right) \\
& \mathcal{C}_{4}^{(1)}=\partial \Omega+\frac{1}{2}\left(e \partial_{e}+\bar{e} \partial_{\bar{e}}+f \partial_{f}+\bar{f} \partial_{\bar{f}}\right)+g \partial_{g}+\bar{g} \partial_{\bar{g}} \\
& \mathcal{C}_{5}^{(1)}=\mathcal{C}_{5} \\
& \mathcal{C}_{6}^{(1)}=\partial \partial_{f}+\bar{\partial} \partial_{\bar{f}}-\frac{1}{2} \Omega \partial_{\bar{f}}-\frac{3}{2} \sigma \partial_{f}-\frac{1}{2} \partial_{\sigma} \partial_{\bar{f}}+\frac{1}{\sqrt{2}}\left(e \partial_{g}+\bar{e} \partial_{\bar{g}}\right) \\
& \mathcal{C}_{7}^{(1)}=\partial \partial_{e}+\bar{\partial} \partial_{\bar{e}}-\frac{1}{2} \Omega \partial_{\bar{e}}+\frac{3}{2} \sigma \partial_{e}+\frac{1}{2} \partial_{\sigma} \partial_{\bar{e}}-\frac{1}{\sqrt{2}}\left(f \partial_{g}+\bar{f} \partial_{\bar{g}}\right) \\
& \mathcal{C}_{8}^{(1)}=\partial \partial_{g}+\bar{\partial} \partial_{\bar{g}}-\Omega \partial_{\bar{g}}+\frac{1}{\sqrt{2}}\left(\partial_{e} \partial_{\bar{f}}-\partial_{\bar{e}} \partial_{f}\right)
\end{aligned}
$$

where $\Omega$ was defined in eq. (27). As for the above $S l(2, \mathbf{R})$-case we have again dropped $\delta(0)$ or $\delta^{\prime}(0)$-type divergences. We will continue to do so without further warning.

The strategy now is to perform successive changes of variables such that at each step there is one constraint $C_{a}^{(i)} \Psi_{i}=0$ that only involves one functional derivative and hence can be easily integrated (as $\mathcal{C}_{1}$ above). Let

$$
\begin{gathered}
e=e^{\varphi / 2+\psi}, \bar{e}=e^{\varphi / 2+\psi} \beta, \\
f=e^{\varphi / 2+\gamma-\psi}, \bar{f}=e^{\varphi / 2+\gamma-\psi} \alpha, \\
g=e^{\varphi}, \bar{g}=e^{\varphi} \mu .
\end{gathered}
$$

We will see that $\mu$ plays the role of the Beltrami differential and $\varphi$ that of the (chiral)

$\dagger$ Note that e.g. $\mathcal{C}_{6}$ translates into $\mathcal{C}_{6}^{(1)}+\frac{\omega}{\sqrt{2} g} \mathcal{C}_{2}^{(1)}$ but since $\mathcal{C}_{2}^{(1)} \Psi_{1}=0$ was already required separately it is enough to impose $\mathcal{C}_{6}^{(1)} \Psi_{1}=0$ with $\mathcal{C}_{6}^{(1)}$ independent of $\omega, \partial_{\omega}$. Similar remarks hold for $\mathcal{C}_{7}^{(1)}$ and $\mathcal{C}_{8}^{(1)}$. 
Liouville field. Thus $g$ and $\bar{g}$ (not $e$ and $\bar{e}$ ) are zweibein components. Then $\mathcal{C}_{4}^{(1)}$ becomes

$$
\mathcal{C}_{4}^{(1)}=\partial_{\varphi}+\partial(\mu \partial \varphi-\bar{\partial} \varphi+L), \quad L=\partial \mu+\frac{e^{\gamma}}{\sqrt{2}}(\beta-\alpha)
$$

so that $\mathcal{C}_{4}^{(1)} \Psi_{1}=0$ is readily solved by

$$
\begin{aligned}
& \Psi_{1}=\exp \left(-\frac{k}{2 \pi} S_{2}\right) \Psi_{2} \\
& S_{2}=\int \mathrm{d}^{2} z\left[-\frac{1}{2} \partial \varphi \bar{\partial} \varphi+\mu\left(\frac{1}{2}(\partial \varphi)^{2}-\partial^{2} \varphi\right)+\frac{e^{\gamma}}{\sqrt{2}}(\beta-\alpha) \partial \varphi\right]
\end{aligned}
$$

with $\Psi_{2}$ independent of $\varphi$. Again $S_{2}$ looks like a chiral version of the Liouville action with $\mu$ being the Beltrami differential. Note that as compared with the $S l(2, \mathbf{R})$-case, there is an extra coupling of the chiral Liouville field $\varphi$ to the $\alpha$ and $\beta$-fields. Using eqs. (31), the other constraints translate into $\mathcal{C}_{a}^{(2)} \Psi_{2}=0, a=2,3,5,6,7,8$ with

$$
\begin{aligned}
\mathcal{C}_{2}^{(2)}= & \frac{1}{\sqrt{2}} e^{-\gamma}\left(\alpha \partial_{\alpha}-\partial_{\gamma}-\mu \partial_{\alpha}\right)+\frac{1}{2} \partial_{\sigma}-\frac{1}{2} L+\beta \partial \psi+\partial \beta-\frac{3}{2} \beta \sigma-\bar{\partial} \psi, \\
\mathcal{C}_{3}^{(2)}= & -\frac{1}{\sqrt{2}} e^{-\gamma}\left(\beta \partial_{\beta}-\partial_{\psi}-\partial_{\gamma}-\mu \partial_{\beta}\right)-\frac{1}{2} \partial_{\sigma}-\frac{1}{2} L \\
& +\alpha \partial \gamma-\alpha \partial \psi+\partial \alpha+\frac{3}{2} \alpha \sigma-\bar{\partial} \gamma+\bar{\partial} \psi \\
\mathcal{C}_{5}^{(2)}= & -\frac{1}{2} \partial_{\psi}+\frac{1}{3} \partial \partial_{\sigma}-\bar{\partial} \sigma, \\
\mathcal{C}_{6}^{(2)}= & \left(\partial+\partial \psi-\partial \gamma-\frac{3}{2} \sigma\right)\left(\partial_{\gamma}-\alpha \partial_{\alpha}\right)+\left(\bar{\partial}+\bar{\partial} \psi-\bar{\partial} \gamma-\frac{1}{2} L\right) \partial_{\alpha}-\frac{1}{2} \partial_{\sigma} \partial_{\alpha} \\
& +\frac{e^{\gamma}}{\sqrt{2}}\left(\beta \partial_{\mu}-\partial_{\gamma}-\mu \partial_{\mu}-\frac{1}{2} \partial_{\psi}-\partial L\right) \\
\mathcal{C}_{7}^{(2)}= & \left(\partial-\partial \psi+\frac{3}{2} \sigma\right)\left(\partial_{\gamma}+\partial_{\psi}-\beta \partial_{\beta}\right)+\left(\bar{\partial}-\bar{\partial} \psi-\frac{1}{2} L\right) \partial_{\beta}+\frac{1}{2} \partial_{\sigma} \partial_{\beta} \\
& -\frac{e^{\gamma}}{\sqrt{2}}\left(\alpha \partial_{\mu}-\partial_{\gamma}-\mu \partial_{\mu}-\frac{1}{2} \partial_{\psi}-\partial L\right), \\
\mathcal{C}_{8}^{(2)}= & e^{\gamma}(\bar{\partial}-L) \partial_{\mu}-e^{\gamma} \partial\left(\partial_{\gamma}+\mu \partial_{\mu}+\frac{1}{2} \partial_{\psi}\right)-e^{\gamma} \partial^{2} L \\
& +\frac{1}{\sqrt{2}} \partial_{\alpha}\left(\partial_{\gamma}+\partial_{\psi}-\beta \partial_{\beta}\right)-\frac{1}{\sqrt{2}} \partial_{\beta}\left(\partial_{\gamma}-\alpha \partial_{\alpha}\right) .
\end{aligned}
$$

Changing variables further:

$$
e^{\gamma}=\frac{r+\Sigma}{\sqrt{2}}, \sigma=\frac{r-\Sigma}{2}, \beta=e^{-\psi} \hat{\beta}+\mu, \alpha=\frac{\sqrt{2}}{r+\Sigma} e^{\psi} \hat{\alpha}+\mu
$$


we can solve first $\mathcal{C}_{2}^{(2)} \Psi_{2}=0$ and then $\mathcal{C}_{3}^{(3)} \Psi_{3}=0$ with

$$
\begin{aligned}
\Psi_{2}= & \exp \left(-\frac{k}{2 \pi} S_{3}\right) \Psi_{3}=\exp \left(-\frac{k}{2 \pi}\left(S_{3}+S_{4}\right)\right) \Psi_{4}, \\
S_{3}=\int \mathrm{d}^{2} z\left[\left(\frac{1}{4} e^{-\psi} \hat{\beta}+\frac{3}{8} \mu\right) \Sigma^{2}\right. & \\
& \left.+\Sigma\left(\frac{1}{2} \partial \mu-\frac{3}{4} r \mu+\mu \partial \psi-\bar{\partial} \psi+e^{-\psi} \partial \hat{\beta}-e^{-\psi} r \hat{\beta}+\frac{e^{\psi} \hat{\alpha}}{2 \sqrt{2}}\right)\right] \\
S_{4}=\int \mathrm{d}^{2} z & {\left[\psi(\bar{\partial} r-r \partial \mu-\mu \partial r)-\frac{1}{\sqrt{2}} e^{\psi}\left(2 \partial \hat{\alpha}+\frac{5}{2} r \hat{\alpha}\right)+\frac{1}{2} e^{-\psi} r\left(2 \hat{\beta}-\frac{5}{2} r \hat{\beta}\right)\right] . }
\end{aligned}
$$

The remaining constraints $\mathcal{C}_{a}^{(2)} \Psi_{2}=0, a=5, \ldots 8$ then are replaced by $\mathcal{C}_{a}^{(4)} \Psi_{4}=$ $0, a=5, \ldots 8$ with $^{\star}$

$$
\begin{aligned}
\mathcal{C}_{5}^{(4)} & =\hat{\beta} \partial_{\hat{\beta}}-\hat{\alpha} \partial_{\hat{\alpha}}-\frac{2}{3} \partial \partial_{r}+2 \bar{\partial} r+\frac{1}{3} \partial^{2} \mu-\frac{3}{2} \partial(r \mu) \\
\mathcal{C}_{6}^{(4)} & =\left(\bar{\partial}-\mu \partial-\frac{5}{4} \partial \mu+\frac{3}{8} r \mu\right) \partial_{\hat{\alpha}}-\frac{1}{2} \partial_{\hat{\alpha}} \partial_{r}+\frac{1}{\sqrt{2}}\left(\hat{\beta} \partial_{\mu}+2 \partial^{2} \hat{\beta}-\frac{5}{2} \hat{\beta} \partial r-6 r \partial \hat{\beta}+\frac{45}{8} r^{2} \hat{\beta}\right) \\
\mathcal{C}_{7}^{(4)} & =\left(\bar{\partial}-\mu \partial-\frac{7}{4} \partial \mu-\frac{3}{8} r \mu\right) \partial_{\hat{\beta}}+\frac{1}{2} \partial_{\hat{\alpha}} \partial_{r}-\frac{1}{\sqrt{2}}\left(\hat{\alpha} \partial_{\mu}+2 \partial^{2} \hat{\alpha}+\frac{7}{2} \hat{\alpha} \partial r+6 r \partial \hat{\alpha}+\frac{45}{8} r^{2} \hat{\alpha}\right) \\
\mathcal{C}_{8}^{(4)} & =(\bar{\partial}-\mu \partial-2 \partial \mu) \partial_{\mu}-\left(\frac{1}{2} \hat{\alpha} \partial+\frac{3}{2} \partial \hat{\alpha}\right) \partial_{\hat{\alpha}}-\left(\frac{1}{2} \hat{\beta} \partial+\frac{3}{2} \partial \hat{\beta}\right) \partial_{\hat{\beta}} \\
& +r \partial \partial_{r}-\partial^{3} \mu-\frac{3}{4} r \bar{\partial} r+\frac{1}{2} \partial \bar{\partial} r-\frac{1}{2} \partial^{2}(r \mu)
\end{aligned}
$$

At this point we change our polarization by performing a Laplace transformation:

$$
\Psi_{4}(r, \hat{\alpha}, \hat{\beta}, \mu)=\int \mathcal{D} \hat{r} \exp \left(\frac{k}{2 \pi} \int \mathrm{d}^{2} z r(z) \hat{r}(z)\right) \hat{\Psi}_{4}(\hat{r}, \hat{\alpha}, \hat{\beta}, \mu)
$$

(Thus the original wave functional $\Psi$ is given by a (functional integral) convolution of the transform of $\exp \left(-\frac{k}{2 \pi} \sum_{i=1}^{4} S_{i}\right)$ and of $\hat{\Psi}_{4}$. $)$ This Laplace transform has the effect of exchanging the roles of $r$ and $\partial_{r}$ :

$$
r \rightarrow \partial_{\hat{r}}, \quad \partial_{r} \rightarrow-\hat{r}
$$

* We have subtracted $\frac{9}{4} r \mathcal{C}_{5}^{(4)}$ from the result one obtains for $\mathcal{C}_{8}^{(4)}$ : one is always free to replace the constraints by some linear combinartion of them. 
We also perform an ultimate change of variables and set

$$
\hat{r}=R-\frac{1}{2} \partial \mu
$$

Then, the new constraints are $\hat{\mathcal{C}}_{a}^{(4)} \hat{\Psi}_{4}=0, a=5, \ldots 8$ with

$$
\begin{aligned}
\hat{\mathcal{C}}_{5}^{(4)}= & \left(\bar{\partial}-\frac{3}{4} \mu \partial-\frac{3}{4} \partial \mu\right) \partial_{R}+\frac{1}{2}\left(\hat{\beta} \partial_{\hat{\beta}}-\hat{\alpha} \partial_{\hat{\alpha}}\right)+\frac{1}{3} \partial R \\
\hat{\mathcal{C}}_{6}^{(4)}= & \left(\bar{\partial}-\mu \partial-\frac{3}{2} \partial \mu+\frac{1}{2} R\right) \partial_{\hat{\alpha}}+\frac{3}{8} \mu \partial_{R} \partial_{\hat{\alpha}} \\
& +\frac{1}{\sqrt{2}}\left(\hat{\beta} \partial_{\mu}+\frac{45}{8} \hat{\beta} \partial_{R} \partial_{R}-6 \partial \hat{\beta} \partial_{R}-3 \hat{\beta} \partial \partial_{R}+2 \partial^{2} \hat{\beta}\right) \\
\hat{\mathcal{C}}_{7}^{(4)}= & \left(\bar{\partial}-\mu \partial-\frac{3}{2} \partial \mu-\frac{1}{2} R\right) \partial_{\hat{\beta}}-\frac{3}{8} \mu \partial_{R} \partial_{\hat{\beta}} \\
& +\frac{1}{\sqrt{2}}\left(-\hat{\alpha} \partial_{\mu}-\frac{45}{8} \hat{\alpha} \partial_{R} \partial_{R}-6 \partial \hat{\alpha} \partial_{R}-3 \hat{\alpha} \partial \partial_{R}-2 \partial^{2} \hat{\alpha}\right), \\
\hat{\mathcal{C}}_{8}^{(4)}= & (\bar{\partial}-\mu \partial-2 \partial \mu) \partial_{\mu}-\partial^{3} \mu-\left(\frac{1}{2} \hat{\alpha} \partial+\frac{3}{2} \partial \hat{\alpha}\right) \partial_{\hat{\alpha}}-\left(\frac{1}{2} \hat{\beta} \partial+\frac{3}{2} \partial \hat{\beta}\right) \partial_{\hat{\beta}} \\
& -\partial R \partial_{R}-\frac{3}{8} \bar{\partial}\left(\partial_{R} \partial_{R}\right) .
\end{aligned}
$$

We observe the symmetry $\hat{\alpha} \leftrightarrow \hat{\beta}, \hat{\mathcal{C}}_{6}^{(4)} \leftrightarrow \hat{\mathcal{C}}_{7}^{(4)}$ (up to some sign changes). We also note that on the hypersurface in field space $R=\hat{\alpha}=\hat{\beta}=0$ and in the subspace of the Hilbertspace where $\partial_{\tau} \hat{\Psi}_{4}=\partial_{\hat{\alpha}} \hat{\Psi}_{4}=\partial_{\hat{\beta}} \hat{\Psi}_{4}=0, \hat{\mathcal{C}}_{5}^{(4)}, \hat{\mathcal{C}}_{6}^{(4)}$ and $\hat{\mathcal{C}}_{7}^{(4)}$ vanish while $\hat{\mathcal{C}}_{8}^{(4)} \hat{\Psi}_{4}=0$ reduces to the conformal Ward identity (remember that $\partial_{\mu} \equiv-\frac{2 \pi}{k} \frac{\delta}{\delta \mu}$ ):

$$
\left((\bar{\partial}-\mu \partial-2 \partial \mu) \frac{\delta}{\delta \mu}+\frac{k}{2 \pi} \partial^{3} \mu\right) \hat{\Psi}_{4}=0
$$

cf. eq . (20). In fact, the full set of constraints $\hat{\mathcal{C}}_{a}^{(4)} \hat{\Psi}_{4}=0, a=5, \ldots 8$ constitutes the Ward identities of some extension of the conformal algebra, namely the $W_{3}^{(2)}$. algebra. We will show this by working out the more familiar operator product expansions (OPEs) that follow from these Ward identities.

Let us sketch how one obtains the corresponding OPEs. Since by eq. (40) $\hat{\Psi}_{4}$ is identified with a conformal block, $\frac{\delta}{\delta \mu(z)}$ acting on $\hat{\Psi}_{4}$ produces an insertion of $-\frac{1}{\pi} T(z)$, $T$ being the stress tensor. Similarly $\frac{\delta}{\delta R(z)}$ produces an insertion of an operator $U(z)$, 
etc. Thus we have

$$
\begin{aligned}
\partial_{\mu} \leftrightarrow \frac{2}{k} \tilde{T}(z), \\
\partial_{\hat{\alpha}} \leftrightarrow \frac{2^{3 / 4}}{k} G^{-}(z), \\
\partial_{\hat{\beta}} \leftrightarrow \frac{2^{3 / 4}}{k} G^{+}(z), \\
\partial_{R} \leftrightarrow \frac{1}{k} U(z),
\end{aligned}
$$

where we put a tilde over $T$ since the true stress tensor will be a slight modification of $\tilde{T}$. Now consider e.g. the Ward identity $\hat{\mathcal{C}}_{5}^{(4)} \hat{\Psi}_{4}=0$. This reads

$$
\bar{\partial} U-\frac{3}{4} \partial(\mu U)+2^{-1 / 4} \hat{\beta} G^{-}-2^{-1 / 4} \hat{\alpha} G^{+}+\frac{k}{3} \partial R=0
$$

which should be thought of as an equality valid when inserted into some correlation function. This equation is taken at the point $z$. Now multiply with $\frac{1}{w-z}$, integrate $\int \mathrm{d}^{2} z$ and use $\bar{\partial}_{z} \frac{1}{w-z}=-\pi \delta^{(2)}(w-z)$ to obtain

$$
-\pi U(w)=\int \mathrm{d}^{2} z\left[\frac{3}{4} \frac{\mu(z) U(z)}{(w-z)^{2}}+2^{-1 / 4} \frac{\hat{\beta}(z) G^{+}(z)}{w-z}-2^{-1 / 4} \frac{\hat{\alpha}(z) G^{-}(z)}{w-z}-\frac{k}{3} \frac{R(z)}{(w-z)^{2}}\right] .
$$

Next take $\frac{\delta}{\delta R(z)}$ and set $\mu=\hat{\alpha}=\hat{\beta}=R=0$ (note that $\mu=0$ is the conformal gauge):

$$
U(w) U(z)=-\frac{2 k}{3} \frac{1}{(w-z)^{2}}
$$

If instead we take $\frac{\delta}{\delta \mu(z)}$ and set $\mu=\hat{\alpha}=\hat{\beta}=R=0$ :

$$
U(w) \tilde{T}(z)=\frac{3}{4} \frac{U(z)}{(w-z)^{2}} \Leftrightarrow \tilde{T}(w) U(z)=\frac{3}{4}\left(\frac{U(z)}{(w-z)^{2}}+\frac{\partial U(z)}{w-z}\right)+\ldots
$$

and similarly:

$$
U(w) G^{ \pm}(z)= \pm \frac{G^{ \pm}(z)}{w-z}
$$

One sees that $U$ will be a conformal spin- 1 field if we define the stress tensor as

$$
T=\tilde{T}-\frac{3}{16 k} U^{2}
$$

Equation (45) then becomes

$$
T(w) U(z)=\frac{U(z)}{(w-z)^{2}}+\frac{\partial U(z)}{w-z}
$$

Proceeding in a similar way with $\hat{\mathcal{C}}_{8}^{(4)}$ one finds that it is indeed $T$ as defined by eq. 
(47) that is the stress tensor: ${ }^{*}$

$$
T(w) T(z)=\frac{c / 2}{(w-z)^{4}}+\frac{2 T(z)}{(w-z)^{2}}+\frac{\partial T(z)}{w-z}, \quad c=6 k
$$

and that $G^{ \pm}$have conformal spin $\frac{3}{2}$ :

$$
T(w) G^{ \pm}(z)=\frac{3}{2} \frac{G^{ \pm}(z)}{(w-z)^{2}}+\frac{\partial G^{ \pm}(z)}{w-z}
$$

Finally, the only new OPEs we get from $\hat{\mathcal{C}}_{6}^{(4)}$ and $\hat{\mathcal{C}}_{7}^{(4)}$ are

$$
\begin{aligned}
& G^{+}(w) G^{-}(z)=-\frac{T(z)+(3 / k) U^{2}(z)}{w-z}+\frac{3 U(z)}{(w-z)^{2}}+\frac{3}{2} \frac{\partial U(z)}{w-z}-\frac{2 k}{(w-z)^{3}} \\
& G^{ \pm}(w) G^{ \pm}(z)=0 .
\end{aligned}
$$

Equations (44), (46) and (48) to (51) are the OPEs of the $W_{3}^{(2)}$-algebra. They exactly correspond to the Poisson bracket algebra as given e.g. in ref. 14 (with $k=-1$ ).

\section{Conclusions}

Quantizing the $S l(3, \mathbf{R})$ Chern-Simons theory in the large (i.e. unconstrained) Hilbert space one has to impose 8 conditions on the physical wave functionals $\Psi$. Choosing a certain polarization (i.e. 8 fields on which $\Psi$ depends) we have been able to explicitly solve 4 of these physical state conditions. This was done by an ansatz $\Psi=\exp \left(-\frac{k}{2 \pi} \sum_{i=1}^{4} S_{i}\right) \Psi_{4}$ where the $S_{i}$ are explicitly determined and $\Psi_{4}$ only depends on 4 fields. The remaining 4 physical state conditions become functional differential equations for $\Psi_{4}$ which turned out to be equivalent to the Ward identities of the $W_{3}^{(2)}$-algebra (implying the OPEs). We might have chosen a different polarization and obtained the Ward identities of the $W_{3}^{(1)} \equiv W_{3}$-algebra. This will be described elsewhere.

* Note that for consistency one should not include double-contraction terms between $U^{2}(z)$ and $U^{2}(w)$ since they correspond to short-distance regularization effects which we have dropped throughout this paper. 


\section{ACKNOWLEDGEMENTS}

An important part of this work was done while I was visiting the Department of Theoretical Physics of the University of Geneva. I am very grateful to Professors R. Gatto and H. Ruegg for their kind hospitality.

\section{REFERENCES}

[1] A.B. Zamolodchikov, TMP 65 (1985) 1205;

V.A. Fateev and A.B. Zamolodchikov, Nucl. Phys. B280[FS18] (1987) 644.

[2] F. Bais, P. Bouwknegt, M. Surridge and K. Schoutens, Nucl. Phys. B304 (1988) 348,371 .

[3] M. Bershadsky and H. Ooguri, Comm. Math. Phys. 126 (1989) 49.

[4] J. Balog, L. Feher, P. Forgacs, L. O'Raifeartaigh and A. Wipf, Phys. Lett. B227 (1989) 214, Ann. Phys. 203 (1990) 76.

[5] K. Yamagishi, Phys. Lett. B205 (1988) 466;

P. Mathiew, Phys. Lett. B208 (1988) 101;

I. Bakas, Phys. Lett. B213 (1988) 313.

[6] P. Di Francesco, C. Itzykson and J.-B. Zuber, "Classical $W$-algebras", Princeton/Saclay preprint PUPT-1211/S.Ph-T/90-149 (October 1990).

[7] A. Bilal and J.-L. Gervais, Phys. Lett. B206 (1988) 412, Nucl. Phys. B314 (1989) 646, Nucl. Phys. B318 (1989) 579.

[8] A. Bilal, Nucl. Phys. B330 (1990) 399, Int. J. Mod. Phys. A5 (1990) 1881, see also in: Proc. $13^{\text {th }}$ Johns Hopkins Workshop, Florence 1989, L. Lusanna ed. (World Scientific), and in: Proc. Trieste Summer School, July 1989, J.C. Pati et al. eds. (World Scientific).

[9] M. Fukuma, H. Kawai and R. Nakayama, Int. J. Mod. Phys. A6 (1991) 1385;

R. Dijkgraf, E. Verlinde and H. Verlinde, Nucl. Phys. B348 (1991) 435;

J. Goeree, "W-constraints in 2D quantum gravity", Utrecht preprint THU-19 (September 1990).

[10] A. Bilal, V.V. Fock and I.I. Kogan, Nucl. Phys. B359 (1991) 635.

[11] H. Verlinde, Nucl. Phys. B337 (1990) 652.

[12] F.A. Bais, T. Tjin and P. van Driel, "Covariantly coupled chiral algebras", Amsterdam preprint ITFA-90-31 (November 1990). 
[13] M. Bershadsky, "Conformal field theories via Hamiltonian reduction", Princeton preprint IASSNS-HEP-90/44 (1990).

[14] I. Bakas and D.A. Depireux, "A fractional KdV hierarchy", Maryland preprint UMD-PP91-168 (December 1990). 